Internat. J. Math. \& Math. Sci.

Vol. 24, No. 3 (2000) 213-216

S0161171200003847

(C) Hindawi Publishing Corp.

\title{
COMPOSITION OF FUNCTIONS
}

\section{KANDASAMY MUTHUVEL}

(Received 26 March 1999 and in revised form 11 August 1999)

\begin{abstract}
We prove that if $f$ and $g$ are functions from the reals into the reals such that the composition of $g$ with $f$ is continuous and $f$ is both Darboux and surjective, then $g$ is continuous. We also prove that continuous and Darboux can be interchanged in the above statement.
\end{abstract}

Keywords and phrases. Darboux functions, continuous functions, composition of functions.

2000 Mathematics Subject Classification. Primary 26A15; Secondary 54C30.

1. Introduction. Throughout the paper, $f$ and $g$ are functions from the reals into the reals. The composition of $g$ with $f$ is denoted by $g \circ f$. It is easy to find examples to show that if $g \circ f$ is continuous, then neither $f$ nor $g$ is continuous. The problem becomes more interesting if some restrictions are put on $f$. For example, a well-known theorem states that if $g \circ f$ is continuous, and $f$ is both continuous and surjective, then $g$ is continuous. However, it follows from our example that if "continuous" is replaced by "open" or "Darboux" in the above statement, then the new statements are no longer true. In this paper, we prove some interesting results concerning composition of functions. For example, we generalize the above well-known result by showing that if $g \circ f$ is continuous, and $f$ is both Darboux and surjective, then $g$ is continuous. This also improves [3, Theorem 3] which states that "if $g \circ f$ is continuous, $f$ and $g$ are Darboux, and $f$ is surjective, then $g$ is continuous." We also prove that continuous and Darboux can be interchanged in the above statement of our result, i.e., if $g \circ f$ is Darboux and $f$ is both continuous and surjective, then $g$ is Darboux.

DEFINITION 1.1. A function $f$ from the reals into the reals is called Darboux if it has the intermediate value property, i.e., if $a$ and $b$ are real numbers with $a<b$ and $f(a) \neq f(b)$, then for any real number $y$ between $f(a)$ and $f(b)$ there exists a real number $x$ between $a$ and $b$ such that $f(x)=y$.

Note that every continuous function is Darboux, but not every Darboux function is continuous.

THEOREM 1.2. Let $f$ and $g$ be functions from the reals into the reals and let $f$ be surjective.

(i) If $g \circ f$ is continuous and $f$ is Darboux, then $g$ is continuous.

(ii) If $g \circ f$ is Darboux and $f$ is continuous, then $g$ is Darboux.

Proof of (i). Assume, to the contrary, that the function $g$ is discontinuous at a real number $a$, from the right. Since $f$ is surjective, there are real numbers $b$ and $c$ such that $f(b)=a$ and $f(c)>a$. We consider the following two cases. 
CASE $1(c<b)$. Let $X=\{x<b: f(x)>a\}$ and let $d$ be the supremum of $X$. Then $c \leq d \leq b$. If $f(d)>a$, then, since $f$ is Darboux and $a=f(b)<f(d)$, there exists a real number $t$ in the nonempty open interval $(d, b)$ such that $f(t)>a$, which contradicts that $d$ is the supremum of $X$. Hence, $f(d) \leq a$ and $d \notin X$. Consequently, there exists a sequence $\left(d_{n}\right)$ in $X$ such that $d_{n}<d$ for all $n$ and $\left(d_{n}\right)$ converges to $d$. (*) Since $g$ is discontinuous at $a$ from the right, there exists a sequence $\left(x_{n}\right)$ converging to $a$ such that $x_{n}>a$ for all $n$ and no subsequence of $\left(g\left(x_{n}\right)\right)$ converges to $g(a)$. Note that $\left(x_{n}\right)$ converges to $a$, for all $n, a<x_{n}$ and $a<f\left(d_{n}\right)$. Hence, there exists a subsequence $\left(y_{n}\right)$ of $\left(x_{n}\right)$ such that $a<y_{n}<f\left(d_{n}\right)$. Since $f$ is Darboux and $f(d) \leq a<y_{n}<f\left(d_{n}\right)$, we have $y_{n}=f\left(t_{n}\right)$ for some $t_{n}$ in the nonempty open interval $\left(d_{n}, d\right)$. Hence, $\left(t_{n}\right)$ converges to $d$. Since $g \circ f$ is continuous at $d$, the sequence $\left((g \circ f)\left(t_{n}\right)\right)=\left(g\left(y_{n}\right)\right)$ converges to $(g \circ f)(d)$. Consequently, by $(*), g(f(d)) \neq g(a)$. Since $f\left(d_{n}\right)>a>$ $f(d)$ for all $n$ and $f$ is Darboux, there exists a real number $s_{n}$ in the nonempty open interval $\left(d_{n}, d\right)$ such that $f\left(s_{n}\right)=a$. Hence, $\left(s_{n}\right)$ converges to $d$. Again, since $g \circ f$ is continuous at $d$ and $(g \circ f)\left(s_{n}\right)=g\left(f\left(s_{n}\right)\right)=g(a)$, we have $g(a)=g(f(d))$, which is impossible.

CASE $2(c>b)$. Let $X=\{x>b: f(x)>a\}$ and let $d$ be the infimum of $X$. As shown in Case 1 , it is not hard to show that this case is impossible.

Thus $g$ is continuous at $a$ from the right. Similarly, $g$ is continuous at $a$ from the left. Thus $g$ is continuous.

ProOF OF (ii). Let $a$ and $b$ be real numbers and $a<b$. Suppose $y$ is a real number between $g(a)$ and $g(b)$. Since $f$ is surjective, $f^{-1}(a)$ and $f^{-1}(b)$ are nonempty sets. Let $c \in f^{-1}(a)$ and $d \in f^{-1}(b)$. Let $H$ be the intersection of the set $f^{-1}(a)$ and the closed interval having $c$ and $d$ as end points, and let $K$ be the intersection of the set $f^{-1}(b)$ and the closed interval having $c$ and $d$ as end points. Since $f$ is continuous, $H$ and $K$ are disjoint nonempty compact sets. It is easy to see that there exist $h \in H$ and $k \in K$ such that $|h-k|=\operatorname{dist}(H, K)=\min \{|s-t|: s \in H$ and $t \in K\}$. Since $(g \circ f)(h)=g(f(h))=g(a),(g \circ f)(k)=g(b), y$ is between $g(a)$ and $g(b)$, and $g \circ f$ is Darboux, there exists a real number $r$ between $h$ and $k$ such that $(g \circ f)(r)=y$. The proof is complete if we show that $a<f(r)<b$. To prove this, suppose, to the contrary, that $f(r) \leq a$. Because $f$ is Darboux and $f(r) \leq a<f(k), a=f(x)$ for some $x$ between $r$ and $k$, or $x=r$. Consequently, $x \in H$ and $|x-k|<|h-k|$, which contradicts that $|h-k|=\operatorname{dist}(H, K)$. Hence, $f(r)>a$. Similarly, $f(r)<b$. Because $a<f(r)<b$ and $g(f(r))=y, g$ is Darboux.

Note that [1, Theorem 1] is the following corollary.

COROLLORY 1.3. If $g \circ f$ is continuous and $f$ is both continuous and surjective, then $g$ is continuous.

[3, Theorem 3] states that if $g \circ f$ is continuous, $f$ is surjective, and $f$ and $g$ are Darboux, then $g$ is continuous. Note that our theorem shows that " $g$ is Darboux" is not needed in [3, Theorem 3].

REMARK 1.4. If "continuous" and "Darboux" are interchanged in statement (i) of Theorem 1.2, we get statement (ii) of Theorem 1.2. If "Darboux" is replaced by "open" 
in statement (i) of Theorem 1.2, then the new statement "If $g \circ f$ is continuous, and $f$ is both open and surjective, then $g$ is continuous" is true. Proof of this proposition is straightforward. If "Darboux" is replaced by "open" in statement (ii) of Theorem 1.2, then the new statement "If $g \circ f$ is open, and $f$ is both continuous and surjective, then $g$ is open" is true. This is an exercise in [2]. It follows from the following examples that if "continuous" is replaced by "Darboux" or "open" in statements (i) and (ii) of Theorem 1.2, then the new statements are no longer true.

EXAMPLE 1.5. There are functions $f$ and $g$ from the reals into the reals such that $f$ is surjective, $f$ is both open and Darboux, and $g \circ f$ is both open and Darboux, but $g$ is neither open nor Darboux. For, let $f: \mathbb{R} \rightarrow \mathbb{R}$ be a function that maps every nonempty open interval onto $\mathbb{R}$. Such a function can be easily constructed by transfinite induction. Let $g(1)=2, g(2)=1$, and $g(x)=x$ when $1 \neq x \neq 2$.

It is interesting to compare the following example with (i) and (ii) of Theorem 1.2.

EXAMPLE 1.6. There are functions $f$ and $g$ from the reals into the reals such that $g$ is surjective, $g \circ f$ is continuous, and $g$ is both Darboux and open, but $f$ is neither Darboux (hence $f$ is not continuous) nor open.

For, let $g: \mathbb{R} \rightarrow \mathbb{R}$ be a function that maps every nonempty open interval onto $\mathbb{R}$. Then there exist two distinct real numbers $a$ and $b$ such that $g(a)=g(b)=0$. Let $f: \mathbb{R} \rightarrow \mathbb{R}$ be a function with two-element range $\{a, b\}$.

REMARK 1.7. For an injective function $g$, it is not hard to show that the statements $g$ is continuous, $g$ is open, and $g$ is Darboux are equivalent. Using the fact that $g^{-1}$ is continuous and the composition of continuous functions is continuous, Darboux functions is Darboux, and open functions is open, we have the following.

Let $f$ and $g$ be functions from the reals into the reals and let $g$ be injective.

(i) If $g \circ f$ is continuous and $g$ is Darboux, then $f$ is continuous.

(ii) If $g \circ f$ is Darboux and $g$ is Darboux, then $f$ is Darboux.

(iii) If $g \circ f$ is open and $g$ is open, then $f$ is open.

EXAMPLE 1.8. There are functions $f$ and $g$ from the reals into the reals such that $g \circ f$ is both open and continuous, and $g$ is continuous, but $f$ is neither Darboux nor open.

For, let $f(x)=x+1$ when $x \geq-1$ and $f(x)=x$ when $x<-1$. Let $g(x)=-x^{2}-x$ when $x<0$ and $g(x)=x$ when $x \geq 0$. Then $f$ is neither Darboux nor open, because

$$
f((-2,0))=(-2,-1) \cup[0,1) .
$$

However, if $f$ and $g$ are functions from the reals into the reals such that $g \circ f$ and $g$ are both open and continuous, then $f$ is open and continuous. This follows from Remark 1.7 and the fact that if $g$ is both open and continuous, then $g$ is injective (otherwise $g$ has a local extreme value on some open interval and consequently $g$ is not open).

AcKNowLEDGEMENT. I would like to thank the referee for the useful comments on the original version of the paper. 


\section{REFERENCES}

[1] K. Ciesielski and S. B. Nadler, Jr., An absorption property for the composition of functions, Real Anal. Exchange 18 (1992/93), no. 2, 420-426. MR 94f:54027. Zbl 791.54020.

[2] J. Dugundji, Topology, Allyn and Bacon Inc., Boston, Mass., 1978. MR 57\#17581.

[3] R. Kellum, Compositions of Darboux-like functions, Real Anal. Exchange 23 (1997/98), no. 1, 211-216. MR 99b:26003.

KANDASAMy MuthuVel: DePaRTMENT OF MATHEMATICS, UniVersity of Wisconsin OSHKOSH, OSHKoSH, Wisconsin 54901-8601, USA

E-mail address: muthuve1@vaxa.cis.uwosh.edu 


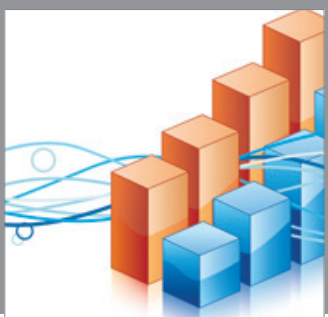

Advances in

Operations Research

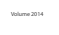

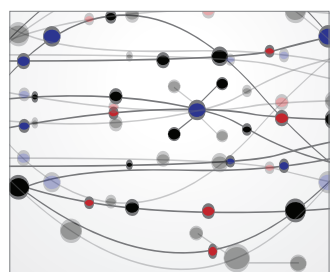

\section{The Scientific} World Journal
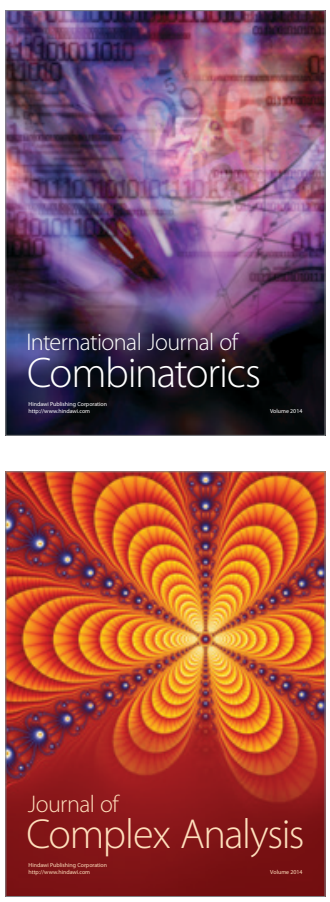

International Journal of

Mathematics and

Mathematical

Sciences
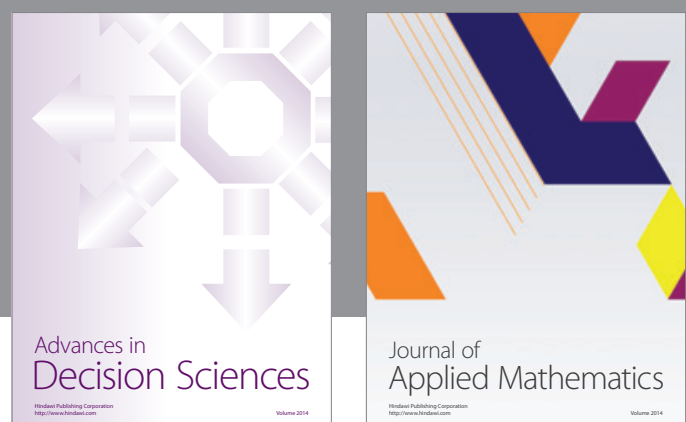

Journal of

Applied Mathematics
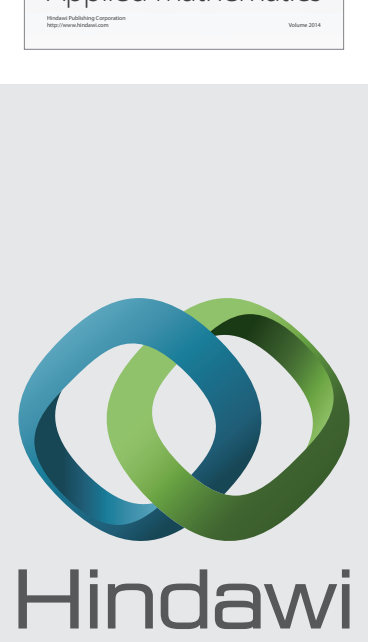

Submit your manuscripts at http://www.hindawi.com
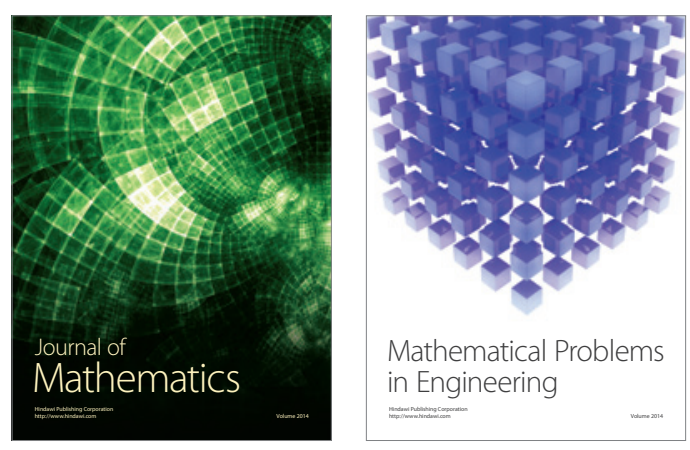

Mathematical Problems in Engineering
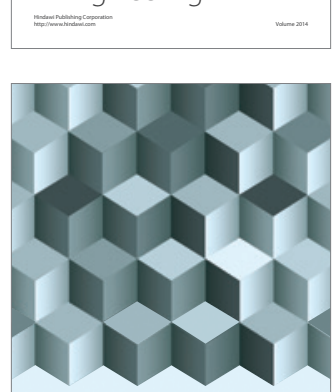

Journal of

Function Spaces
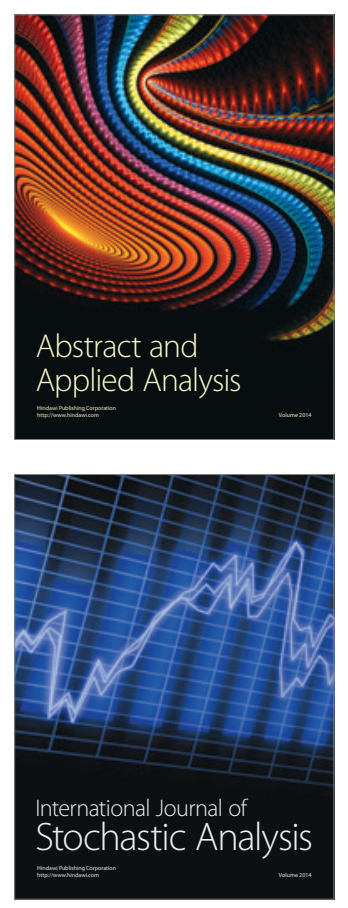

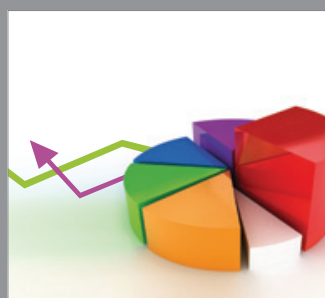

ournal of

Probability and Statistics

Promensencen
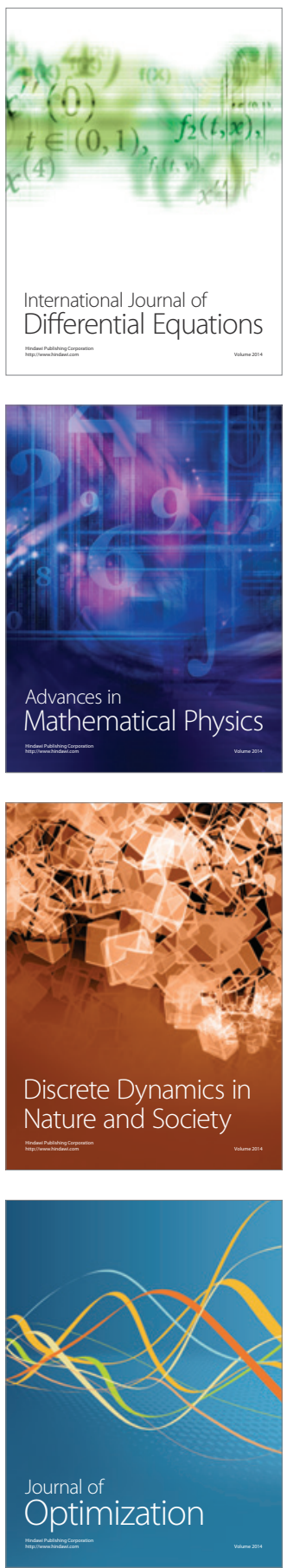